\title{
Higher-Order Lithography: Double-Deprotected Chemically Amplified Photoresists (DD-CAMP)
}

\author{
Deanna Soucie', William Earley ${ }^{1}$, Kenji Hosoi $^{2}$, Arata Takahashi², Takashi Aoki ${ }^{2}$, \\ Brian Cardineau ${ }^{1}$, Koichi Miyauchi ${ }^{2}$, and Robert Brainard ${ }^{1 *}$ \\ ${ }^{1}$ SUNY Polytechnic Institute, Colleges of Nanoscale Science and Engineering, Albany, NY \\ ${ }^{2}$ Central Glass, Chiyoda-ku, Tokyo, Japan \\ *RBrainard@SUNYPoly.edu
}

\begin{abstract}
The synthesis and lithographic evaluation of 193-nm and EUV photoresists that utilize a higher-order reaction mechanism of deprotection is presented. Unique polymers utilize novel blocking groups that require two acid-catalyzed steps to be removed. When these steps occur with comparable reaction rates, the overall reaction should be higher order ( $\leq$ 1.85). The LWR of these resists is plotted against PEB time for a variety of compounds to acquire insight into the effectiveness of the proposed higher-order mechanisms. Evidence acquired during testing of these novel photoresist materials supports the conclusion that higher-order reaction kinetics leads to improved LWR vs. control resists. Keywords: DD-CAMP, Photoresist, EUV, 193 nm, Kinetics, Higher order
\end{abstract}

\section{Introduction}

For the past two decades, the microelectronics industry has maintained Moore's law with the assistance of positive-tone Chemically AMplified Photoresists (CAMP, Fig. 1A-C) [1,2]. This technology has been the driving force behind successes in DUV (248 nm), ArF (193 nm), and EUV $(13.5 \mathrm{~nm})$ lithography. Utilization of CAMP with these lithographic imaging technologies involves a well-known process flow: Exposure of the resist causes the PAG to undergo a photochemical reaction, producing catalytic acid in concentrations that are approximately proportional to the absorbed dose (Fig. 1A). During the postexposure bake (PEB), the acid catalyzes the transformation of the polymer-bound esters to polymer-bound carboxylic acids $\left((\mathrm{P}) \mathrm{CO}_{2} \mathrm{H}\right.$ or $\mathrm{CA}$, Fig. 1). The conversion of the protected ester to the carboxylic acid is first-order in catalytic acid. Therefore, light intensity is approximately proportional to catalytic acid concentration, which is approximately proportional to $\mathrm{CA}$ concentration (Fig. 1D and E). Furthermore, if the aerial image is approximately a sine wave, then the concentration of carboxylic acid groups in the film [CA] will be approximately a sine wave (Fig. 1E) [3]. For the purposes of this paper, we refer to this conventional, first-order CAMP chemistry as singly-deprotected CAMP, or SD-CAMP.

Robert Bristol [3], among others [4,5], has proposed that higher-order responses to light by a photoresist system could provide a number of lithographic benefits by essentially squaring the information provided by the aerial image and thereby enabling double-exposure produced at relaxed pitches [3]. Fabrication of a CAMP photoresist exhibiting a higher-order mechanism would result in the distribution of carboxylic acid resembling a sine squared wave (Fig. 2). In addition to the possibility of using these resists in double-exposure processes [3], these higher-order photoresists have the potential to produce patterns with improved resolution, line edge roughness (LER), and line width roughness (LWR) as a result of the steeper chemical gradient at the line edge (Fig. 3) [6].

Several approaches have been taken previously to develop higher-order photoresist systems including two-photon PAGs and contrastenhancement layers. Two-photon PAGs require two photon interactions to produce the catalytic acid $[7,8]$. In these systems, carboxylic acid concentration is proportional to catalytic acid concentration, while the catalytic acid 
(A) PAG $\stackrel{\text { hv }}{\longrightarrow} \mathrm{H}^{+}+\mathrm{A}^{-}+$Byproducts

(B)

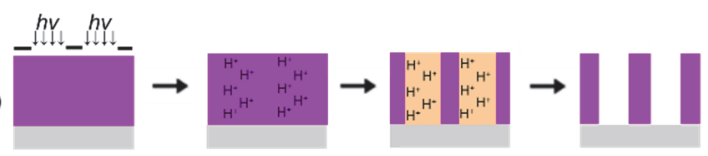

(C)

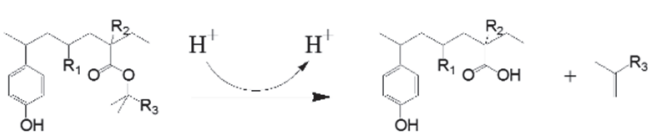

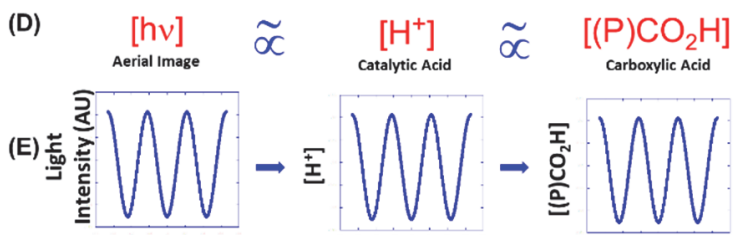

Fig. 1. (A) Decomposition of photoacid generator. (B) Process flow for a positively-toned photoresist. (C) Mechanism of positively-toned photoresist reaction. (D) Relation of light intensity in a photoresist to catalytic acid and carboxylic acid concentrations; $(\mathrm{P})=$ polymer fragment. (E) Graphical representation of the relationship between light intensity, catalytic acid, and carboxylic acid concentrations for equal lines and spaces.

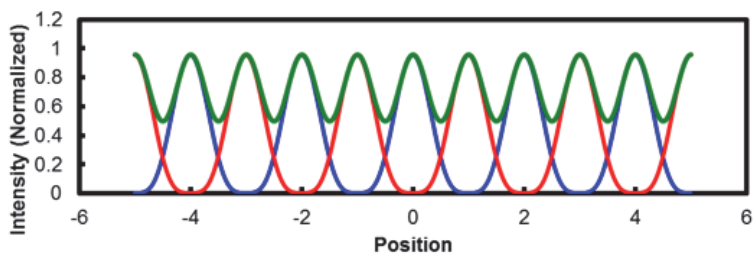

Fig. 2. Double exposure lithography produces two sine wave shaped acid concentration profiles; the sum of these two first-order sine waves will result in the same dose everywhere. "Squaring the aerial image" will allow the two exposures to be combined.

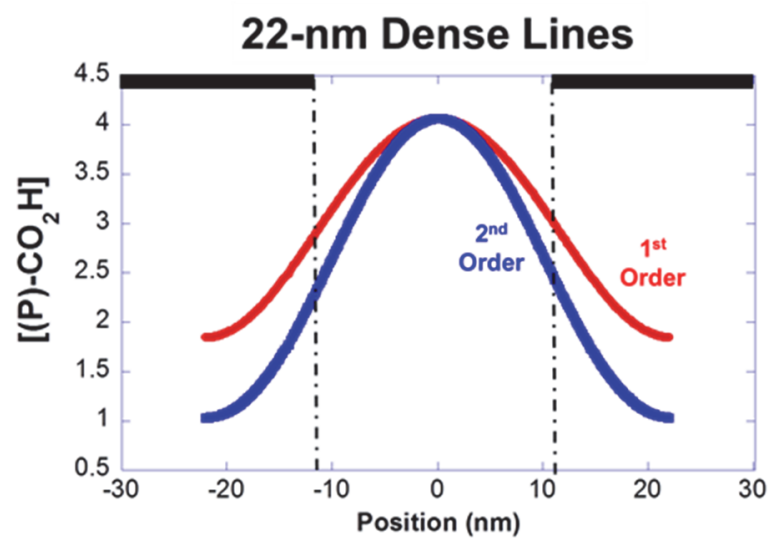

Fig. 3. Carboxylic acid concentration resulting from SD-CAMP first-order and DD-CAMP higher-order kinetics. concentration, while the catalytic acid concentration is exponentially proportionate to light intensity. These PAGs have been demonstrated successfully at higher wavelengths of light (700-1000 nm). However, they are not known to work at $193 \mathrm{~nm}(\mathrm{ArF})$ and $13.5 \mathrm{~nm}$ (EUV), could require two different wavelengths of light, and may have a sensitivity loss on the order of $10^{4}-10^{6}$. Contrast-enhancement layers are photo-bleachable materials that are coated on top of the photoresist. Initially, these layers are opaque, but become progressively more transparent as they are exposed to light [9]. While these materials demonstrate improve-ments in depth of focus and resolution, they exhibit a loss in photospeed of roughly an order of magnitude.

Here, we present a new photoresist system designed to produce higher-order kinetics called, Double-Deprotected Chemically AMplified Photoresists (DD-CAMP). Our approch is a logical extension of our group's design of acid amplifiers [10-13], in which specifically-designed blocking groups protect sulfonic acids. In DDCAMP, however, our specially-designed blocking groups protect polymer-bound carboxylic acids that provide the solubility switches in these positive-tone resists. These new blocking groups are composed of a body and a trigger (BT) which require two acid-catalyzed steps for deprotection (Fig. 4). The trigger must be pulled in the first acid-deprotection in order for the body to be removed in the second. In this work, we explore variations in DD-CAMP blocking groups by systematically changing the body and trigger that compose the resist, and the effect of higher-order kinetics on photoresist performance.

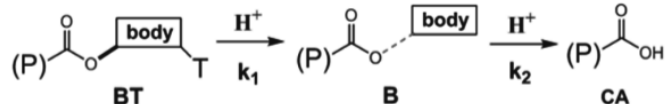

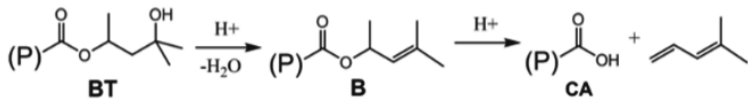

Fig. 4. Basic design of DD-CAMP blocking groups.

\section{Results and discussion}

\subsection{Analysis of reaction kinetics}

One of the tenets in our design of DD-CAMP is that both acid-catalyzed steps must occur at approximately the same rate. If instead, one step is significantly slower than the other (rate-limiting), the overall reaction rate will be first-order in catalytic acid. Therefore, when developing ideas 
for synthesizing specific blocking groups, we considered how the structure of these blocking groups would affect the relative rates of the two acid-deprotection steps.

The simple and familiar rate-law for the acidcatalyzed deprotection of polymer-bound ester in a conventional SD-CAMP resist is shown (Fig. 5). The development of the kinetic rate-law for DDCAMP follows a chapter on sequential reactions in Capellos, Christos and B. Bielski (Fig. 6 and Appendix) [14]. Using this rate law and optimized values for $k_{1}$ and $k_{2}$, we calculated the relative concentrations of three compounds as a function of PEB time: $\mathbf{C A}_{\text {SD }}, \mathbf{B}_{\text {DD }}$ and $\mathbf{C A}_{\text {DD }}$ (Fig. 7). The plot of concentration of carboxylic acid generated using conventional SD-CAMP has the expected shape. The rate of creation of the reaction intermediate, $\mathbf{B}_{\mathbf{D D}}$, initially matches the rate of the creation of $\mathbf{C A}_{\mathbf{S D}}$, but $\mathbf{B}_{\mathbf{D D}}$ is consumed to create $\mathbf{C} \mathbf{A}_{\mathbf{D D}}$. The rate of creation of CA is initially slow, but then accelerates with time. Using a similar approach, we calculated the rate of formation of $\mathbf{C A}_{\mathbf{D D}}$ as a function of $\left[\mathrm{H}^{+}\right]$. We have predicted that the reaction order in $\left[\mathrm{H}^{+}\right]$will reach a maximum of 1.8 when PEB time and the reaction rates of each deprotection, $\mathrm{k}_{1}$ and $\mathrm{k}_{2}$, have been optimized. Linear (Fig. 8A) and log-log plots (Fig. 8B) of $[\mathrm{CA}]$ vs. $\left[\mathrm{H}^{+}\right]$of reactions that are first-order, 1.8order and second-order show that DD-CAMP kinetics are very nearly second-order.

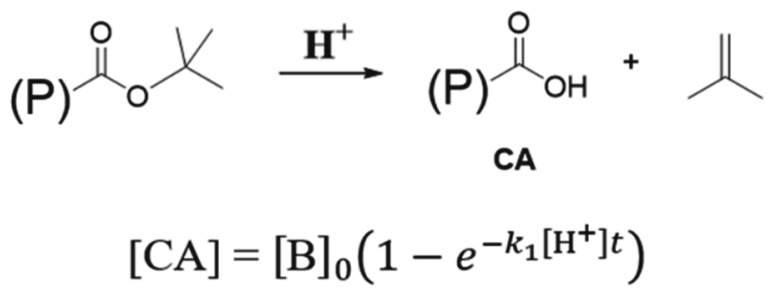

Fig. 5. SD-CAMP reaction mechanism and reaction kinetics.

\subsection{Validation of DD-CAMP reaction pathway hypothesis}

NMR kinetics were used to determine if DDCAMP polymers would follow the predicted twostep, acid-catalyzed decomposition method (Fig. 9). The products shown in Fig. 9 labeled 1, 2 and 3 are the products predicted to form if the predicted mechanism is correct. Products 1 and 2 are isomers of the removed blocking group after the trigger has been pulled and Product 3 is the polymer-bound blocking group with the trigger pulled. Product 4 is the undesired product which would result from the removal of the blocking group without the trigger having been pulled. NMR kinetics showed that the generated products were consistent with those predicted with the proposed DD-CAMP mechanism, and no 4 was detected.

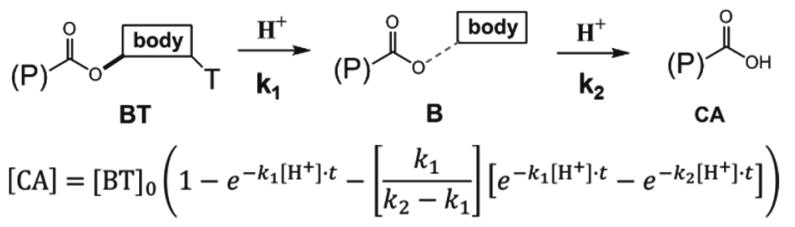

Fig. 6. DD-CAMP reaction mechanism and reaction kinetics.

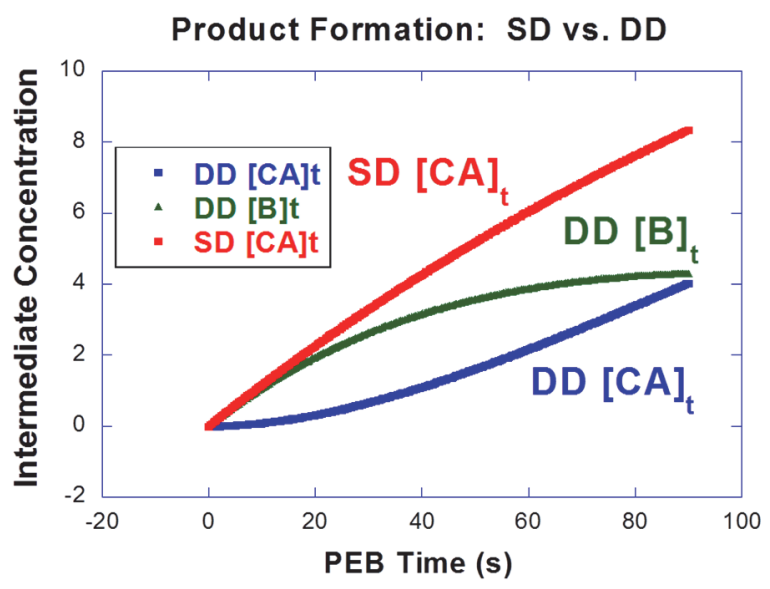

Fig. 7. SD-CAMP and DD-CAMP product concentration as a function of PEB time.

2.3. Validation of higher-order kinetics kypothesis

NMR kinetics were again used to determine the DD-CAMP reaction order. Carboxylic acid concentration was measured as a function of acid concentration. Three NMR samples, each consisting of a solution containing a photopolymer with varying molarity of PAG, $0.05,0.1$, and 0.2 respectively, were exposed to light and the concentration of carboxylic acid produced was measured. Before exposure, carboxylic acid and catalytic acid concentration are zero.

As a control, an SD-CAMP polymer with blocking groups that corresponded to the body group after pulling of the trigger of the DD-CAMP polymer being tested was used (Fig. 10). The control demonstrated a linear relationship between catalytic acid concentration and carboxylic acid concentration. It follows that this reaction is firstorder in catalytic acid concentration. The DDCAMP photopolymer carboxylic acid concentration exhibited an exponential relation to catalytic acid (Fig. 10). The order of the reaction 
was determined by plotting the data from Figure 10 into a log-log plot (Fig. 11). The slope of the resulting line is 1.84 which is the reaction order.

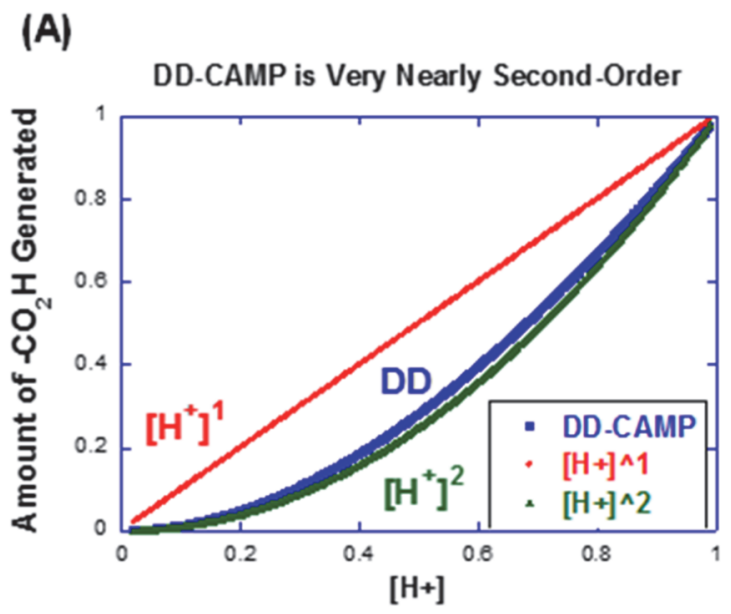

(B)

DD-CAMP is Very Nearly Second-Order

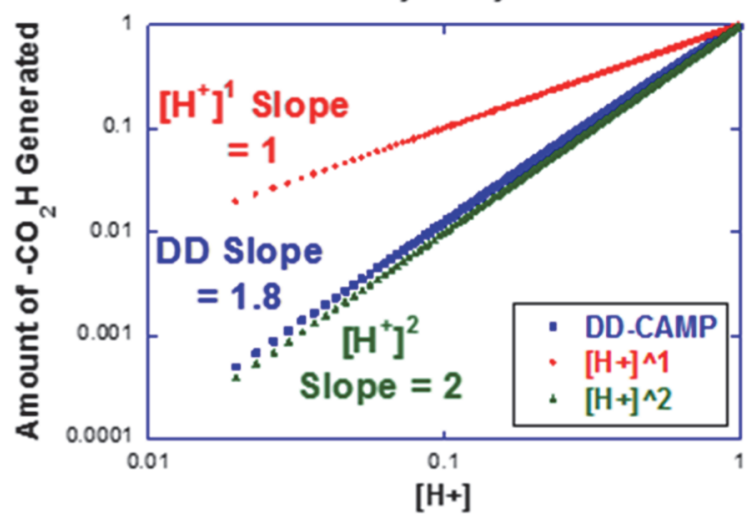

Fig. 8. [CA] vs. $\left[\mathrm{H}^{+}\right]$for first, 1.8 , and second order reactions.

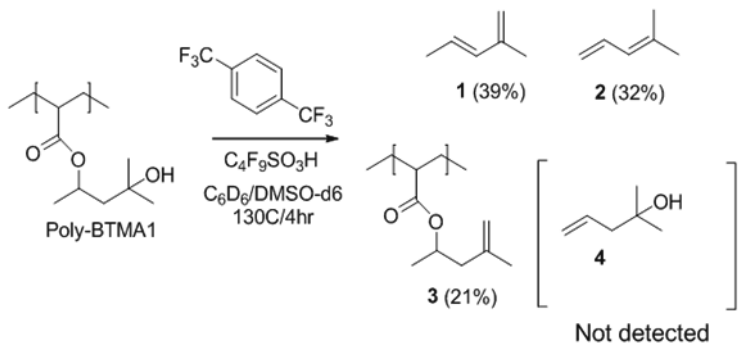

Fig. 9. Results of NMR kinetics performed to determine the decomposition mechanism of DD-CAMP blocking groups.
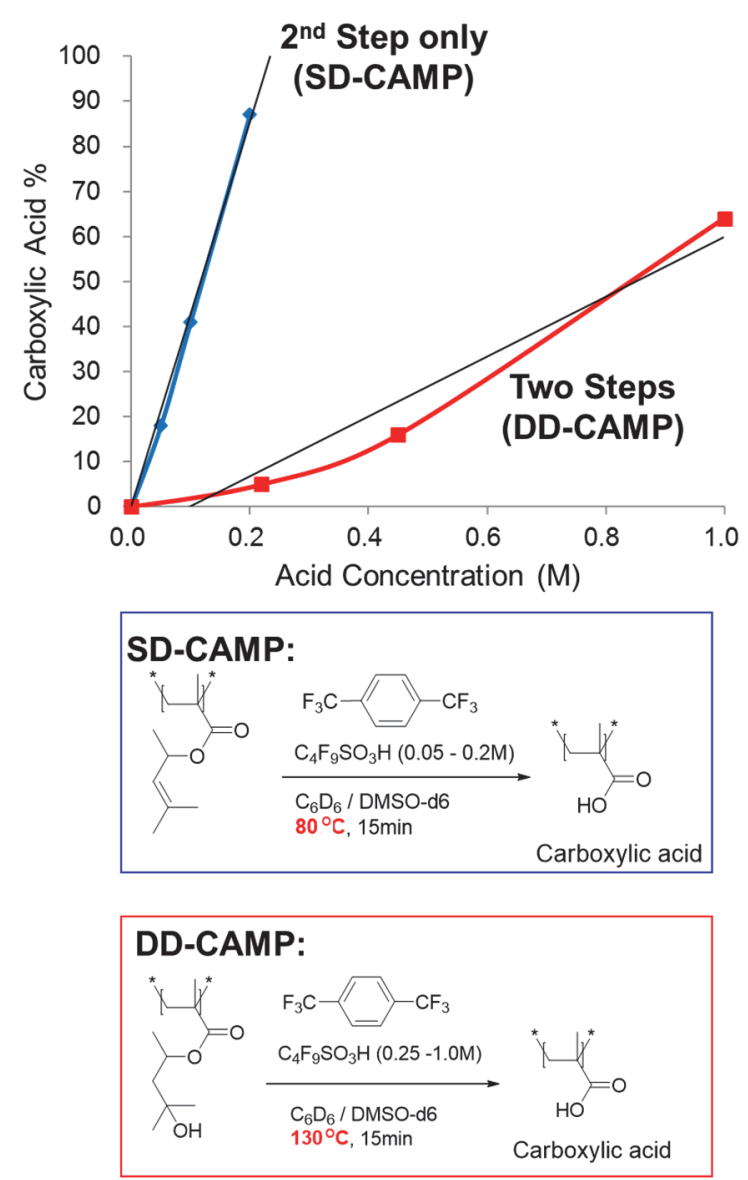

Fig. 10. Method of verifying the first-order SD-CAMP and DD-CAMP carboxylic acid production as a function of catalytic acid concentration.

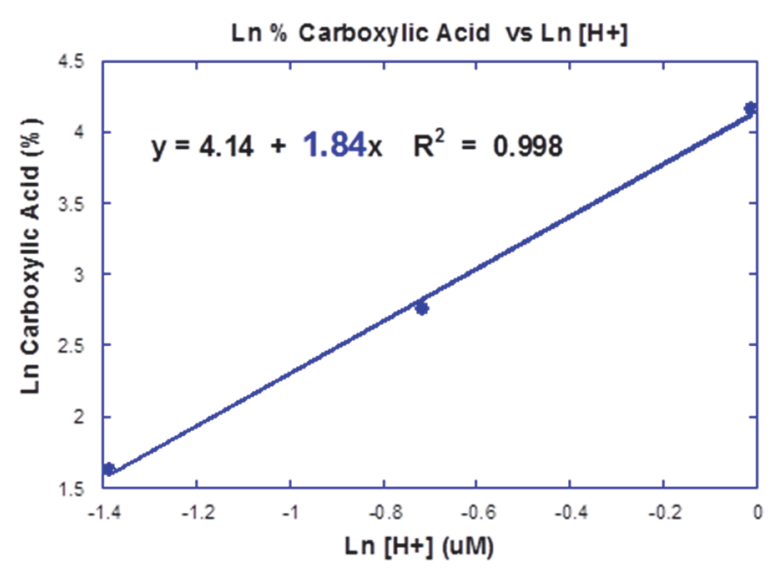

Fig. 11. Log-log plot of DD-CAMP carboxylic acid production.

These higher-order polymers were then combined with PAGs and bases to create EUV or 
193-nm formulations for lithographic testing [1517].

2.4. DD-CAMP synthesis of polymers for use in 193-nm lithography

We have synthesized and evaluated ten novel DD-CAMP monomers for use in 193-nm lithography. The control 193-nm first-order polymer was synthesized from the free-radical polymerization of 3-hydroxy-1-adamantylmethacrylate (HadMA), gamma-butyrolactone methacrylate (GBLMA), and methyl adamantyl methacrylate (MAMA) monomers in a 20/40/40 percent ratio (Fig. 12A). DD-CAMP 193-nm polymer synthesis involves substituting the use of MAMA monomers for DD-CAMP monomers in the free radical polymerization (Fig. 12B). Ten DD-CAMP monomers for 193-nm lithography were synthesized. The structures of seven such monomers are shown in Fig. 12C.

\subsubsection{3-nm imaging experiments}

All DD-CAMP 193-nm photoresists were evaluated using the ASML 1700i 193-nm Immersion Tool at SUNY Polytechnic Institute with dipole illumination, a numerical aperture of 1.2 , and using a sigma value of 0.89 . The photoresist solutions contained 8\% TPS + Nf(PAG) and $1 \%$ TBAH as base [18]. These photoresists were exposed over a range of focus and dose values to determine the optimal exposure conditions. Optimization of the DD-CAMP 193$\mathrm{nm}$ resist made from monomer A1 resulted in maximum image quality which was obtained when dose and focus were $33 \mathrm{~mJ} / \mathrm{cm}^{2}$ and -0.05 , respectively (Fig. 13). The LWR resulting from these exposure conditions was determined with SuMMIT to be $3.7 \mathrm{~nm}$.

To test our hypothesis, the lithographic performance of the DD-CAMP polymer was compared to our SD-CAMP control. The LWR of 193-nm DD-CAMP photoresists varies exponentially with PEB time (Fig. 14). DD-CAMP 193-nm photoresists demonstrate significantly lower LWR when PEB time has been optimized.

\subsection{DD-CAMP polymers for EUV lithography}

We synthesized and evaluated 22 novel DDCAMP monomers for use in EUV lithography. The control EUV first-order polymer is synthesized from the free radical polymerization of hydroxystyrene, styrene, and tert-butyl acrylate monomers in a 20/40/40 percent ratio (Fig. 15A).
DD-CAMP EUV polymer synthesis involves substituting the use of tert-butyl acrylate monomers for DD-CAMP monomers in the free radical polymerization (Fig. 15B). Seventeen EUV DD-CAMP monomers were synthesized, incorporated into polymers and resist formulations were evaluated (Fig. 15C).
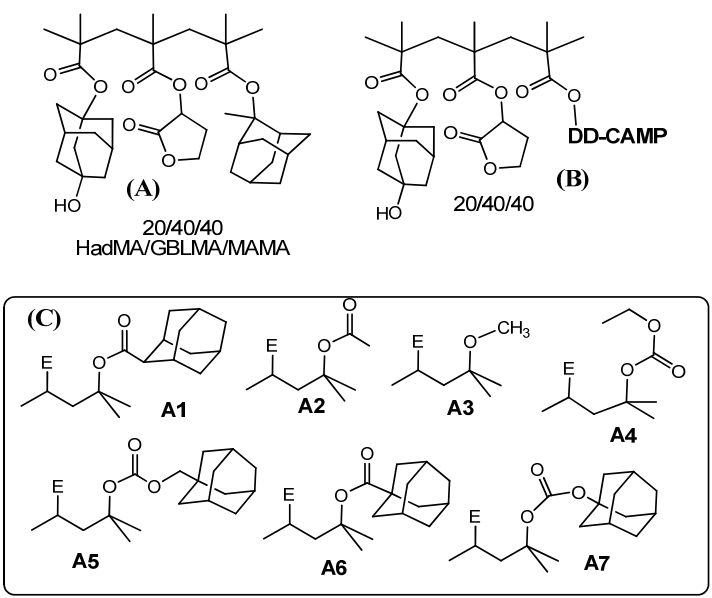

Fig. 12. Structures of (A) SD-CAMP control and (B) DD-CAMP polymers for 193-nm lithography. (C) Seven DD-CAMP monomers for 193-nm lithography where $\mathrm{E}=(\mathrm{P}) \mathrm{CO}_{2-}$.

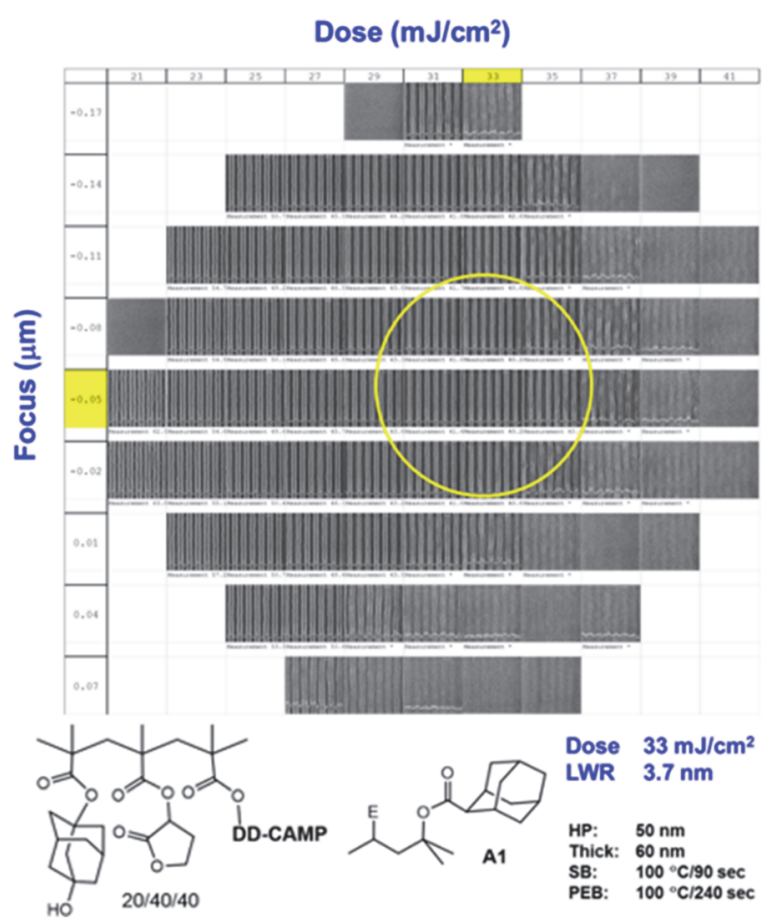

Fig. 13. Lithographic imaging of the DD-CAMP photopolymer made with the DD-CAMP monomer labelled A1. Focus is the $y$ axis component of this graph and dose in $\mathrm{mJ} / \mathrm{cm}^{2}$ is the $\mathrm{x}$ component. 


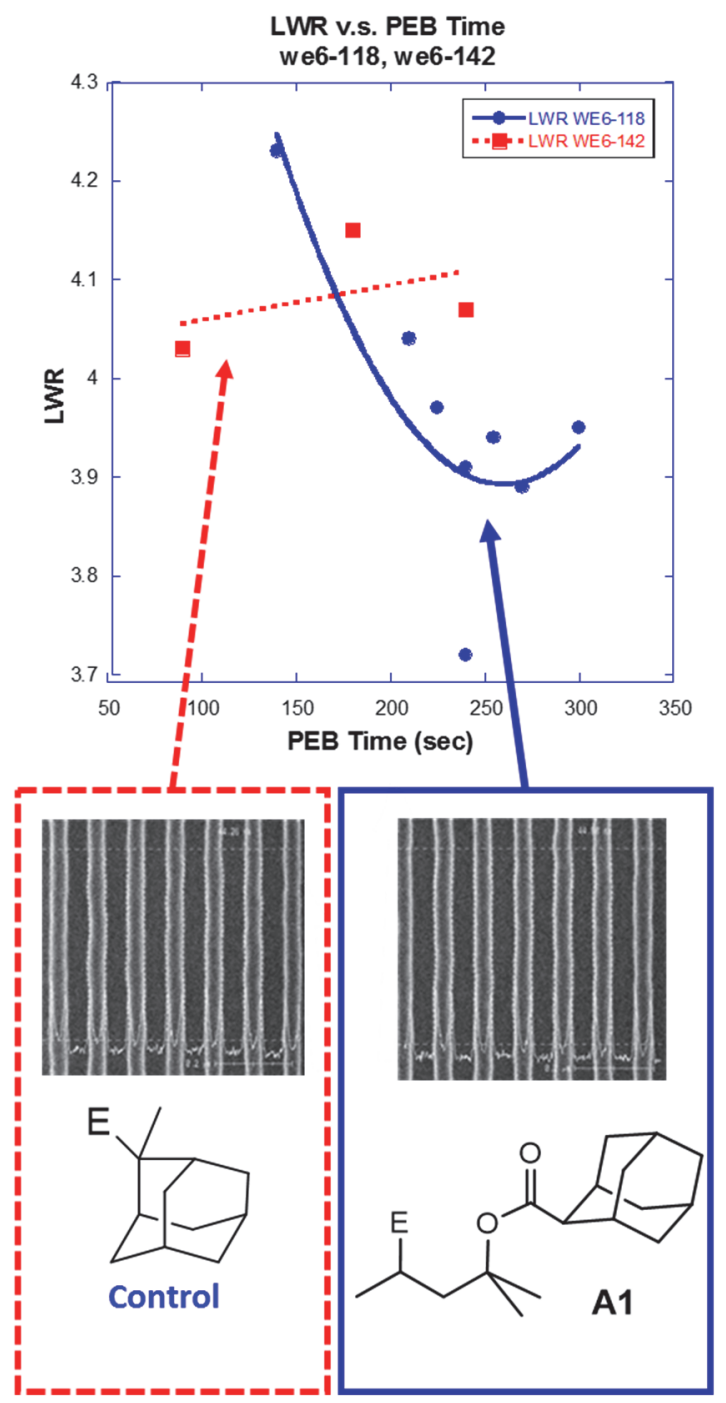

Fig. 14. Comparison of the lithographic performance of DD-CAMP higher-order kinetics photoresists to that of SD-CAMP first-order resists.

\subsubsection{EUV imaging experiments}

Lithographic imaging of DD-CAMP polymers for EUV was performed using the Albany Micro Exposure Tool (AMET) at SUNY Polytechnic Institute on HMDS primed silicon with quadrupole illumination and a numerical aperture of 0.3 . The photoresist solutions contained 8\% TPS+ Nf(PAG) and $1 \%$ TBAH as base. Lithographic imaging results of DD-CAMP EUV photoresist E1 are shown (Fig. 16).

SD-CAMP photoresists have a small variation in LWR with changing PEB time. However, 193$\mathrm{nm}$ and EUV DD-CAMP polymers exhibit an exponential change in LWR for small variations in PEB time. Due to the narrow exposure latitude of DD-CAMP resists, the optimum PEB time must be determined for each higher-order photoresist. PEB time was systematically varied for DD-CAMP resists and LWR measured. Plots of these optimization experiments exhibit parabolic relations between PEB time and LWR (Fig. 17).

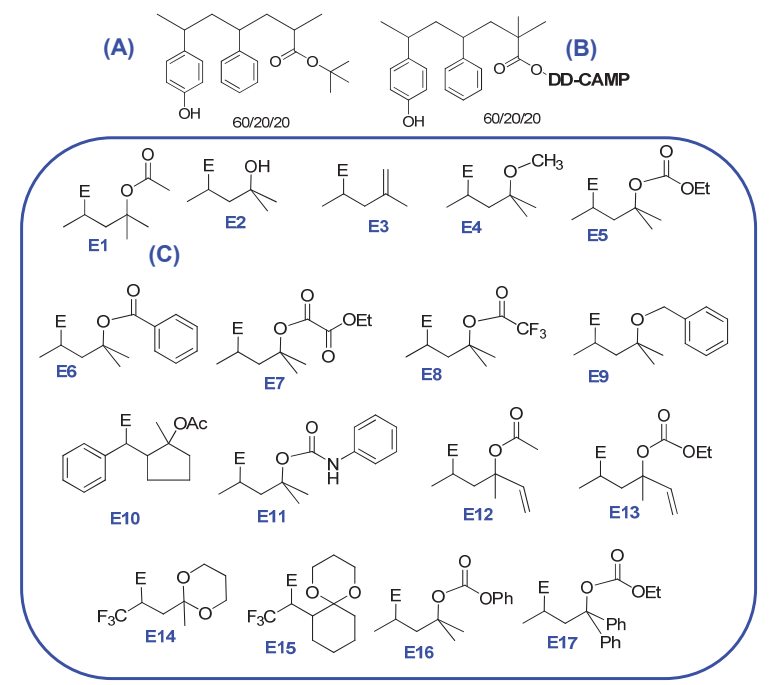

Fig. 15. Structures of (A) SD-CAMP control and (B) DD-CAMP polymers for EUV lithography. (C) Seventeen DD-CAMP monomers for EUV lithography where $\mathrm{E}=(\mathrm{P}) \mathrm{CO}_{2}-$.

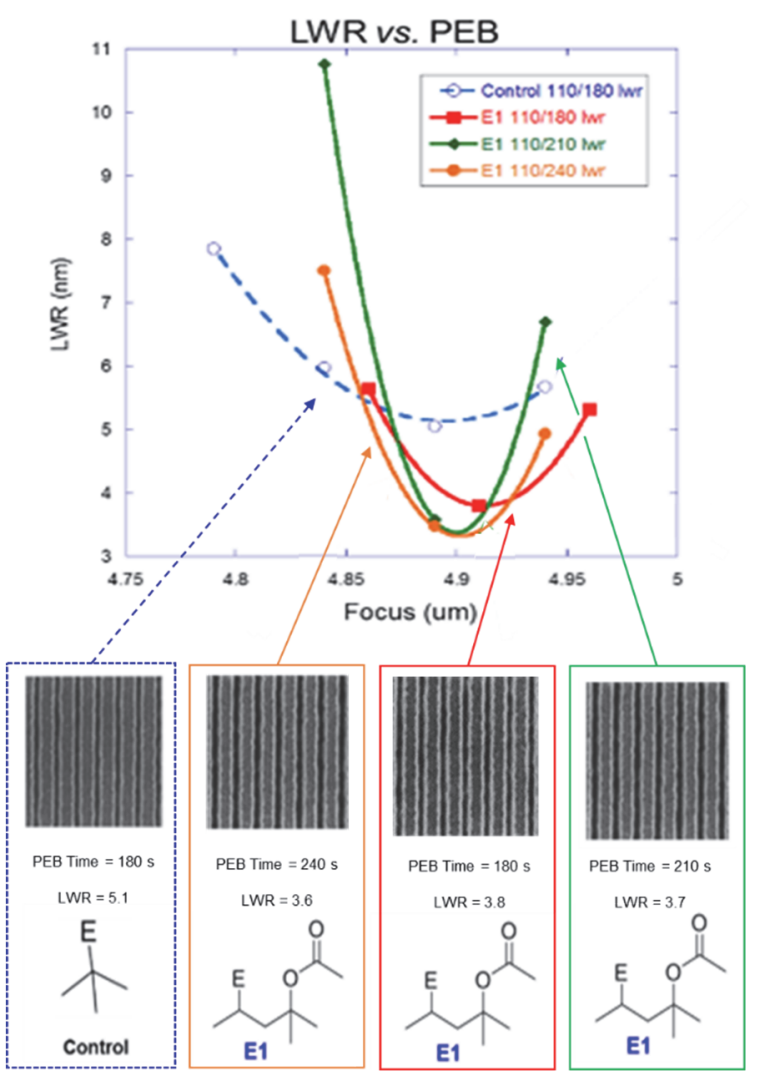

Fig. 16. LWR results for DD-CAMP EUV photoresist E1. 


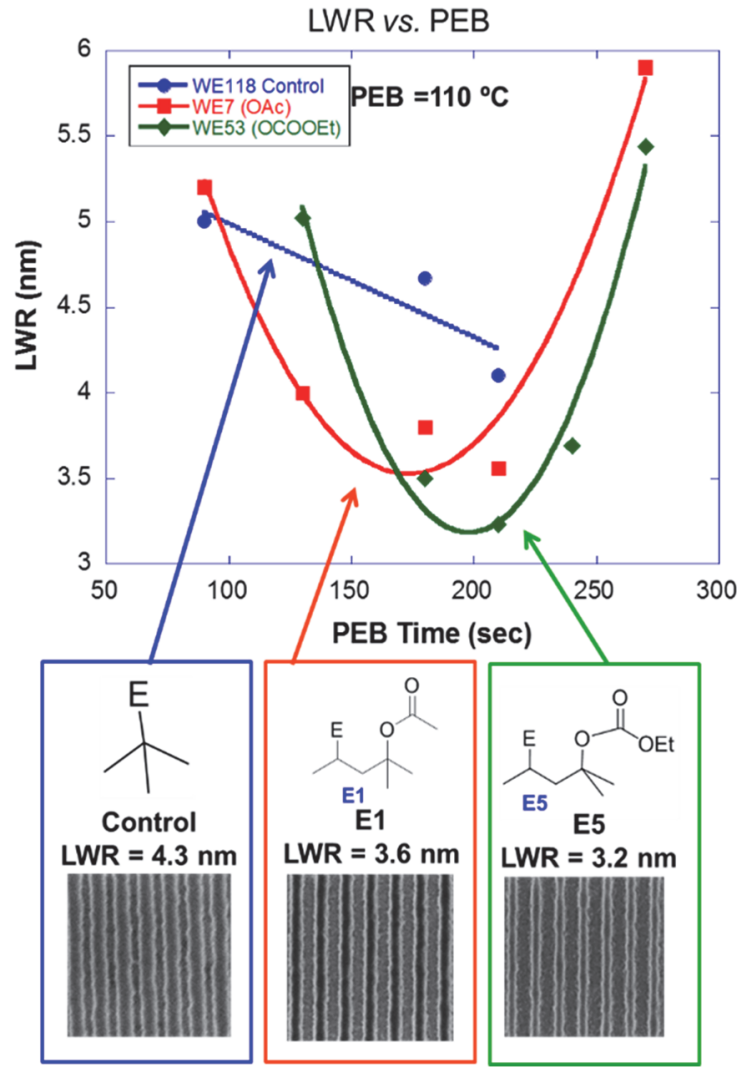

Fig. 17. PEB time optimization tests for EUV DDCAMP resists E1 and E5.

SD-CAMP photoresists have a small variation in LWR with changing PEB time. However, 193$\mathrm{nm}$ and EUV DD-CAMP polymers exhibit an exponential change in LWR for small variations in PEB time. Due to the narrow exposure latitude of DD-CAMP resists, the optimum PEB time must be determined for each higher-order photoresist. PEB time was systematically varied for DD-CAMP resists and LWR measured. Plots of these optimization experiments exhibit parabolic relationships between PEB time and LWR (Fig. 17).

DD-CAMP EUV photoresists demonstrate significantly improved LWR over SD-CAMP control when PEB time and focus are optimized.

\section{Conclusions}

Novel DD-CAMP photopolymers for $193-\mathrm{nm}$ and EUV lithography have been synthesized to produce a higher-order response to photoacid. These DD-CAMP polymers utilize a novel blocking group which consists of a body and a trigger. The first acid-catalyzed step removes the trigger, and the second acid-catalyzed step removes the body, deprotecting the carboxylic acid. Higherorder lithography occurs when the two acidcatalyzed steps have comparable reaction rates. We have synthesized ten DD-CAMP 193-nm monomers and 22 DD-CAMP EUV monomers. Solution phase NMR studies of DD-CAMP polymers indicate higher-order kinetics with an order of 1.84. Lithographic imaging studies of non-optimized resists demonstrate improved LWR when the correct PEB time is selected.

\section{Acknowledgements}

We gratefully acknowledge Central Glass for financial and intellectual support of this work. The authors would like to thank Amrit Narasimhan for their guidance and invaluable feedback, and like to thank Jay Chun, Michael O'Sullivan, Warren Montgomery and Mac Mellish for their assistance in performing the immersion 193-nm resist evaluations.

\section{Appendix}

\section{SD-CAMP Kinetics Derivation Given:}

$$
\begin{aligned}
& \mathrm{B}+\mathrm{H}^{+} \stackrel{\mathrm{k}}{\rightarrow} \mathrm{A}+\mathrm{H}^{+} \\
& \frac{\mathrm{d}[\mathrm{B}]}{\mathrm{dt}}=-\mathrm{k}[\mathrm{B}]\left[\mathrm{H}^{+}\right] \\
& \frac{\mathrm{d}[\mathrm{B}]}{[\mathrm{B}]}=-\mathrm{k}\left[\mathrm{H}^{+}\right] \mathrm{dt}
\end{aligned}
$$

Integration yields:

$$
\begin{aligned}
& \int_{\mathrm{B}_{\mathrm{o}}}^{\mathrm{B}} \frac{\mathrm{d}[\mathrm{B}]}{[\mathrm{B}]}=\int_{0}^{\mathrm{t}}-\mathrm{k}\left[\mathrm{H}^{+}\right] \mathrm{dt} \\
& \ln [\mathrm{B}]-\ln [\mathrm{B}]_{\mathrm{o}}=-\mathrm{k}\left[\mathrm{H}^{+}\right] \mathrm{t} \\
& {[\mathrm{B}]=[\mathrm{B}]_{\mathrm{o}} \mathrm{e}^{-\mathrm{k}\left[\mathrm{H}^{+}\right] \mathrm{t}} \quad \text { Since: }} \\
& {[\mathrm{B}]=[\mathrm{B}]_{\mathrm{o}}-[\mathrm{A}]} \\
& {[\mathrm{B}]=[\mathrm{B}]_{\mathrm{o}} \mathrm{e}^{-\mathrm{k}\left[\mathrm{H}^{+}\right] \mathrm{t}}=\left[\mathrm{B}_{\mathrm{o}}\right]-[\mathrm{A}]} \\
& {[\mathrm{A}]=[\mathrm{B}]_{\mathrm{o}} \mathrm{e}^{-\mathrm{k}\left[\mathrm{H}^{+}\right] \mathrm{t}}}
\end{aligned}
$$

\section{DD-CAMP Kinetics Derivation} Given:

$$
\begin{aligned}
& \mathrm{BT}+\mathrm{H}^{+} \stackrel{\mathrm{k}_{1}}{\rightarrow} \mathrm{B}+\mathrm{H}^{+} \\
& \mathrm{B}+\mathrm{H}^{+} \stackrel{\mathrm{k}_{2}}{\rightarrow} \mathrm{A}+\mathrm{H}^{+} \\
& -\frac{\mathrm{d}[\mathrm{BT}]}{\mathrm{dt}}=-\mathrm{k}_{1}[\mathrm{BT}]\left[\mathrm{H}^{+}\right] \\
& \frac{\mathrm{d}[\mathrm{B}]}{\mathrm{dt}}=\mathrm{k}_{1}[\mathrm{BT}]\left[\mathrm{H}^{+}\right]-\mathrm{k}_{2}[\mathrm{~B}]\left[\mathrm{H}^{+}\right] \\
& \frac{\mathrm{d}[\mathrm{CA}]}{\mathrm{dt}}=\mathrm{k}_{2}[\mathrm{~B}]\left[\mathrm{H}^{+}\right]
\end{aligned}
$$


To simplify, let:

$\mathrm{k}_{1}^{\prime}=\mathrm{k}_{1}\left[\mathrm{H}^{+}\right]$and $\mathrm{k}_{2}^{\prime}=\mathrm{k}_{2}\left[\mathrm{H}^{+}\right]$

Following the first-order calculations and Eqn. 6, Eqn. 12 becomes:

$[\mathrm{BT}]=[\mathrm{BT}]_{\mathrm{o}} \mathrm{e}^{-\mathrm{k}_{1}^{\prime} \mathrm{t}}$

Insert this expression into Eqn. 13 to yield the firstorder differential equation:

$\frac{\mathrm{d}[\mathrm{B}]}{\mathrm{dt}}+\mathrm{k}_{2}^{\prime}[\mathrm{B}]=\mathrm{k}_{1}^{\prime}[\mathrm{BT}]_{\mathrm{o}} \mathrm{e}^{-\mathrm{k}_{1}^{\prime} \mathrm{t}}$

Multiplying both sides by $\mathrm{e}^{\mathrm{k}_{2}^{\prime} \mathrm{t}}$ yields:

$\mathrm{e}^{\mathrm{k}_{2}^{\prime} \mathrm{t}} \frac{\mathrm{d}[\mathrm{B}]}{\mathrm{dt}}+\mathrm{e}^{\mathrm{k}_{2}^{\prime} \mathrm{t}} \mathrm{k}_{2}^{\prime}[\mathrm{B}]=\mathrm{k}_{1}^{\prime}[\mathrm{BT}]_{\mathrm{o}} \mathrm{e}^{-\mathrm{k}_{1}^{\prime} \mathrm{t}} \mathrm{e}^{\mathrm{k}_{2}^{\prime} \mathrm{t}}$

$\mathrm{e}^{\mathrm{k}_{2}^{\prime} \mathrm{t}} \mathrm{d}[\mathrm{B}]+\mathrm{e}^{\mathrm{k}_{2}^{\prime} \mathrm{t}} \mathrm{k}_{2}^{\prime}[\mathrm{B}] \mathrm{dt}=\mathrm{k}_{1}^{\prime}[\mathrm{BT}]_{\mathrm{o}} \mathrm{e}^{\left(\mathrm{k}_{2}^{\prime}-\mathrm{k}_{1}^{\prime}\right) \mathrm{t}}$

Since the left side of Eqn. 19 takes the form of $d(u v)=u d v+v d u$, it is the exact differential of $\mathrm{d}\left(\mathrm{e}^{\mathrm{k}_{2}^{\prime} \mathrm{t}}[\mathrm{B}]\right)$. Eqn. 19 can now be integrated:

$\int_{0}^{[\mathrm{B}]} \mathrm{d}\left(\mathrm{e}^{\mathrm{k}_{2}^{\prime} \mathrm{t}}[\mathrm{B}]\right)=\mathrm{k}_{1}^{\prime}[\mathrm{BT}]_{\mathrm{o}} \int_{0}^{\mathrm{t}} \mathrm{e}^{\left(\mathrm{k}_{2}^{\prime}-\mathrm{k}_{1}^{\prime}\right) \mathrm{t}} \mathrm{dt}$

$\mathrm{e}^{\mathrm{k}_{2}^{\prime} \mathrm{t}}[\mathrm{B}]=\frac{\mathrm{k}_{1}^{\prime}[\mathrm{BT}]_{\mathrm{o}}}{\mathrm{k}_{2}^{\prime}-\mathrm{k}_{1}^{\prime}}\left[\mathrm{e}^{\left(\mathrm{k}_{2}^{\prime}-\mathrm{k}_{1}^{\prime}\right) \mathrm{t}}-1\right]$

Substituting back in the values of $\left[\mathrm{H}^{+}\right]$from Eqn. 15 yields:

$\mathrm{e}^{\mathrm{k}_{2} \mathrm{t}}[\mathrm{B}]=\frac{\mathrm{k}_{1}[\mathrm{BT}]_{\mathrm{o}}}{\mathrm{k}_{2}-\mathrm{k}_{1}}\left[\mathrm{e}^{\left(\mathrm{k}_{2}-\mathrm{k}_{1}\right)\left[\mathrm{H}^{+}\right] \mathrm{t}}-1\right]$

Hence:

$[B]=\frac{\mathrm{k}_{1}[\mathrm{BT}]_{\mathrm{o}}}{\mathrm{k}_{2}-\mathrm{k}_{1}}\left[\mathrm{e}^{-\mathrm{k}_{1}\left[\mathrm{H}^{+}\right] \mathrm{t}}-\mathrm{e}^{\mathrm{k}_{2}\left[\mathrm{H}^{+}\right] \mathrm{t}}\right]$

Mass balance of the three components of the reaction yields:

$[\mathrm{CA}]=[\mathrm{BT}]_{\mathrm{o}}-[\mathrm{BT}]-[\mathrm{B}]$

Substitution of eqns. 16 and 23 into 24 yields:

$$
[\mathrm{CA}]=[\mathrm{BT}]_{\mathrm{o}}-[\mathrm{BT}]_{\mathrm{o}} \mathrm{e}^{-\mathrm{k}_{1}\left[\mathrm{H}^{+}\right] \mathrm{t}}-
$$

$\frac{\mathrm{k}_{1}[\mathrm{BT}]_{\mathrm{o}}}{\mathrm{k}_{2}-\mathrm{k}_{1}}\left(\mathrm{e}^{-\mathrm{k}_{1}\left[\mathrm{H}^{+}\right] \mathrm{t}}-\mathrm{e}^{-\mathrm{k}_{2}\left[\mathrm{H}^{+}\right] \mathrm{t}}\right)$

Which simplifies to:

$$
\begin{aligned}
& \quad[\mathrm{CA}]=[\mathrm{BT}]_{\mathrm{o}}\left(1-\mathrm{e}^{-\mathrm{k}_{1}\left[\mathrm{H}^{+}\right] \mathrm{t}}-\frac{\mathrm{k}_{1}}{\mathrm{k}_{2}-\mathrm{k}_{1}}\right)\left(\mathrm{e}^{-\mathrm{k}_{1}\left[\mathrm{H}^{+}\right] \mathrm{t}}-\right. \\
& \left.\mathrm{e}^{-\mathrm{k}_{2}\left[\mathrm{H}^{+}\right] \mathrm{t}}\right)
\end{aligned}
$$

\section{Notes and References}

1. H. Ito, Adv. Polym. Sci., 172 (2005) 37.

2. H. Ito, IBM J. Res. Dev., 44 (2000) 119.

3. R. Bristol, D. Shykind, S. Kim, Y. Borodovsky, E. Schwartz, C. Turner, G. Masson, K. Min, K. Esswein, and J. M. Blackwell, Proc. SPIE, 7273 (2009) 727307.

4. J. Byers, S. Lee, K. Jen, P. Zimmerman, N. Turro, and C. G. Willson, J. Photopolym. Sci. Technol., 20 (2007) 707.

5. J. S. Petersen, R. T. Greenway, T. Fühner, P. Evanschitzky, F. Shao, and A. Erdmann, Proc. SPIE, 7274 (2009) 72421V.

6. R. Brainard, P. Trefonas, J. Lammers, C. Cutler, J. Mackevich, A. Trefonas, and S. Robertson, Proc. SPIE, 5374 (2004) 74.

7. S. Lee, K. Jen, C.G. Willson, J. Byers, P. Zimmerman, and N. J. Turro, $J$. Micro/Nanolith. MEMS MOEMS, 8 (2009) 011011.

8. S. Lee, J. Byers, K. Jen, P. Zimmerman, B. Rice, N. J. Turro, and C. G. Willson, Proc. SPIE, 6924 (2008) 69242A.

9. B. F. Griffing and P. R. West, Polym. Eng. Sci, 23 (1983) 947.

10. S. Kruger, S. Revuru, C. Higgins, S. Gibbons, D. A. Freedman, W. Yueh, T. R. Younkin, and R. L. Brainard, J. Am. Chem. Soc., 131 (2009) 9862.

11. R. L. Brainard, S. Kruger, C. Higgins, S. Revuru, S. Gibbons, D. Freedman, Y. Wang, and T. Younkin, J. Photopolym. Sci. Technol., 22 (2009) 43.

12. S. A. Kruger, C. Higgins, S. Revuru, S. Gibbons, D. Freedman, and R. L. Brainard, Jpn. J. Appl. Phys., 49 (2010) 041602.

13. S. A. Kruger, C. Higgins, B. Cardineau, T. R. Younkin, and R. L. Brainard, Chem. Mater., 22, (2010) 5609.

14. C. Capellos and B. Bielski, "Kinetics Systems, Mathematical Description of Chemical Kinetics in Solution", Wiley, New York, 1972. 
15. J. F. Cameron, G. Pohlers, and Y. Suzuki, J. Photopolym. Sci. Technol., 15 (2002) 453.

16. K. Nozaki, J. Photopolym. Sci. Technol., 23 (2010) 795.

17. Y. Nishimae, H. Yamato, T. Asakura, and M. Ohwa, J. Photopolym. Sci. Technol., 21 (2008) 377.
18. For the 193-nm imaging experiments, we used ODL-102-100 was used as the underlayer, SHB-A940 L35 the Si Anti Reflective Coating (SiARC), and TCX-041 as a top coat. 